Proof of cubic convergence of the method closely follows the more general discussion of Bodewig. We take $A$ as the true value of the first order root,

$$
f(x)=(x-A) g(x), \quad g(A) \neq 0,
$$

and expand $g(x)$ in a generalized Taylor series, powers of $(x-A)$. Setting $x=a_{n}, b_{n}$ in the series of equations (2) and carrying out the indicated division we obtain

$$
\begin{aligned}
& \frac{x_{n+1}-a_{n}}{a_{n}-A}=-\left[1-\left(x_{n+1}-A\right) \frac{g(A)+g\left(x_{n+1}\right)}{2 g(A)}+P_{1}(x-A)+\cdots\right], \\
& \frac{x_{n+1}-b_{n}}{b_{n}-A}=-\left[1-\left(x_{n+1}-A\right) \frac{g(A)+g\left(x_{n+1}\right)}{2 g(A)}+P_{2}(x-A)+\cdots\right],
\end{aligned}
$$

or, finally,

$$
x_{n+1}-A=\frac{\left(a_{n}-A\right)\left(b_{n}-A\right)}{b_{n}-a_{n}} P(x-A),
$$

where $P(x-A)$ represents a power series in $\left(a_{n}-A\right),\left(b_{n}-A\right),\left(x_{n+1}-A\right)$, quadratic terms and higher.

As a numerical example consider the first root of $x \tan x-1=0$. Taking $a=0.8$, $b=0.9$ equation (4) yields the tabulated value 0.8603 . Two or more applications of Newton's method will involve interpolation if one has only a two place table at hand. Estimates of the magnitude of error involved in interpolation by means of (4) have been found by Hummel and Seebeck [5].

\title{
REFERENCES
}

1. J. K. Stewart, Another variation of Newton's method, Am. Math. Monthly, 58, 331-334 (1951).

2. H. J. Hamilton, A type of variation on Newton's method, Am. Math. Monthly, 57, 517-522 (1950).

3. E. Bodewig, On types of convergence and on the behavior of approximations in the neighborhood of $a$ multiple root of an equation, Q. Appl. Math. 7, 325-333 (1949).

4. P. M. Hummel and C. L. Seebeck, A generalization of Taylor's expansion, Am. Math. Monthly, 56, 243-247 (1949)

5. P. M. Hummel and C. L. Seebeck, A new interpolation formula, Am. Math. Monthly 58, 383-389 (1951).

\section{THE SECOND FUNDAMENTAL THEOREM OF ELECTRICAL NETWORKS*}

\section{By CHARLES SALTZER (Case Institute of Technology)}

1. Introduction. This paper will deal with an extension of the work of W. H. Ingram and C. M. Cramlet ${ }^{1}$ as discussed by J. L. Synge. ${ }^{2}$ In addition it will be shown how their theories fit into a unified theory. The terminology of Synge's paper will be used.

A network may be represented by its Thévenin representation, i.e. by regarding its branches as consisting of impedances in series with constant voltage sources; or, it may be represented by its Norton representation, i.e. by regarding the branches as

*Received May 5, 1952. 
consisting of a constant current generator in parallel with an admittance. In the first representation, the Kirchhoff nodal constraints become homogeneous in the branch currents and permit the introduction of mesh currents and the mesh equations, whereas in the second, the Kirchhoff mesh constraints become homogeneous in the branch voltages and allow the introduction of what $\mathrm{P}$. Le Corbeiller ${ }^{3}$ calls the basic voltages and the nodal equations. The theorem of Ingram and Cramlet which Synge describes as the fundamental theorem of electrical network theory is the fundamental theorem for the Thévenin representation of a network; the dual Norton theorem which is fully on a par with this theorem will be proved independently in this paper, and the relations of the two theorems will be discussed. In addition the nodal equations of G. Kron as given by $\mathrm{P}$. Le Corbeiller will be deduced by a method analogous to that of Synge's. It may be noted that the nodal theorem is much easier to prove than the mesh theorem.

In section (2) we will prove the fundamental theorem for nodal networks and in section (3) we deduce Kron's equations. In section (4) we prove that the nodal equations have a unique solution; and in section (5) we show the relation of the theorems for the Thévenin and Norton representations. In section (2), (3) and (4) we shall consider only the Norton representation of a network.

2. Basic node voltages for connected networks. Let $N$ be the number of nodes: $J_{1}, J_{2}, \cdots J_{N}$; and let $B$ be the number of branches. In addition we assign a direction to each branch and define $E_{k}$ as the voltage rise from the initial point to the terminal point of the $k^{\text {th }}$ branch. A path on the network is defined as a finite sequence of branches such that each branch has one node in common with the preceding branch and the other node in common with the succeeding branch. The first branch is required to have only one node in common with the second and the last branch is required to have only one node in common with its predecessor. For two nodes $J_{p}$ and $J_{q}$ and a path directed from $J_{p}$ to $J_{q}$ we define $V_{p q}$ as the sum of the voltage rises of those branches of the path which have the same sense as the path minus the voltage drops of those branches of the path which have a sense opposite to the sense of the path. We note that for the given path $V_{p q}=-V_{q p}$.

If we consider two paths joining $J_{p}$ and $J_{a}$, these two paths taken in opposite senses form a closed path or mesh. Since there are no voltage sources in the network the sum of the voltage rises around the closed path is zero and thus $V_{p q}$ for one path plus $V_{a p}$ for the other path is zero. It follows from this that $V_{p a}$ is independent of the path. If we select one node say $J_{1}$ as a ground node and define $V_{p}=V_{1 p}(p=2,3, \cdots n)$ then $V_{p}$, the potential relative to the ground, is also independent of the path. We shall call $V_{2}, V_{3}, \cdots, V_{N}$ the basic voltages. Since the voltage rise across a branch from $J_{p}$ to $J_{q}$ is $V_{q}-V_{p}$ we have:

Theorem $I(a)$ If the branch voltages of a network satisfy Kirchhoff's mesh law then there are $N-1$ basic voltages such that the branch voltages can be expressed in terms of them. These basic voltages are the potentials of the nodes relative to the ground node.

In addition if we prescribe $N-1$ arbitrary basic voltages and define the voltage drops across the branches as above then we have:

Therorem $I(b)$ An arbitrary set of $N-1$ basic voltages for a network generates a unique set of branch voltage rises which satisfy Kirchhoff's mesh law.

If $E$ is the column matrix of branch voltage rises $E_{1}, E_{2}, \cdots E_{B}$ then

$$
E_{p}=\sum_{a=2}^{N} A_{p a} V_{a} \quad(p=1,2, \cdots B)
$$


where $A_{p q}$ is defined as follows:

$$
A_{p q}=\left\{\begin{array}{l}
1 \text { if } J_{q} \text { is the terminal node of the } p^{\text {th }} \text { branch } \\
-1 \text { if } J_{q} \text { is the initial node of the } p^{\text {th }} \text { branch } \\
0 \text { if } J_{q} \text { is not on the } p^{\text {th }} \text { branch. }
\end{array}\right.
$$

In matrix notation eq. (2.1) can be written

$$
E=A V
$$

Theorems $I(a)$ and $I(b)$ together may now be stated as:

Theorem II: For any connected network taking any node as the ground node there is a matrix $A$ as in (2.2) such that:

(i) For arbitrary values of the associated basic voltages the branch voltages given by $E=A V$ satisfy Kirchhoff's mesh law on every mesh

(ii) For any set of branch voltages which satisfy Kirchhoff's mesh law on every mesh there is a set of basic voltages $V$ such that

$$
E=A V \text {. }
$$

This theorem is the dual analogue of the "central theorem" of Synge, and can be generalized to apply to linearly independent combinations of nodes. In addition the restriction to networks which are not connected is readily eliminated by the use of ground nodes in each subnetwork.

3. Node equations. If we designate the current source of the $r^{\text {th }}$ branch by $I_{r}$ we can define $B$ branch currents $U_{1}, U_{2} \cdots U_{B}$ each of which consists of the corresponding branch source current and the branch current due to the branch potentials. In matrix notation

$$
U=I-Y E
$$

where $Y$ is the admittance matrix of the network whose elements are the self and mutual admittances of the branches.

We write Kirchhoff's node law as

$$
\sum_{p=1}^{B} F_{a p} U_{p}=0 \quad(q=2,3, \cdots N)
$$

where

$$
F_{a D}=\left\{\begin{array}{c}
1 \text { if } J_{a} \text { is the terminal node of branch } p \\
-1 \text { if } J_{a} \text { is the initial node of branch } p \\
0 \text { if } J_{a} \text { is not on branch } p .
\end{array}\right.
$$

In matrix form this becomes

$$
F U=0 .
$$

If we compare (2.2) and (3.3) we see that $F=A_{t}$ where $A_{t}$ is the transpose of $A$. From 
(3.1) we have

$$
A_{\imath}(I-Y E)=0 .
$$

If we define $I^{\prime}$, the column matrix of basic currents by

$$
I^{\prime}=A_{t} I
$$

we can write (3.5) as

$$
I^{\prime}=A_{t} Y E,
$$

or by (2.3)

$$
I^{\prime}=A_{\imath} Y A V .
$$

Further, if we define $Y^{\prime}$ the basic node admittance matrix by

$$
Y^{\prime}=A_{\imath} Y A,
$$

then (3.7) can be written as

$$
I^{\prime}=Y^{\prime} V .
$$

Equations (2.3), (3.6), (3.8) and (3.9) are the basic equations of Kron's nodal method together with (4.1) below.

4. The existence and uniqueness of the solution of the nodal equations. We show here that if $I=0$ implies $E=0$ then the matrix $Y^{\prime}$ is non-singular, and hence the system of equations (3.9) has a unique solution. If $I=0$ then by (3.6) $I^{\prime}=0$ and (3.9) becomes

$$
Y^{\prime} V=0 .
$$

Since $V=0$ is a solution of (4.1) our assertion will be proved if we show that it is the only solution. But if $I=0 \mathrm{implies}$ that $E=0$ then we must have $V=0$. This is a consequence of the remark that if $E=0$, the basic voltages of the nodes on any path are all equal, and since the network is connected and at least one node is connected to the ground node, the basic voltages of all the nodes are zero. Therefore $Y^{\prime}$ is non-singular and from (3.9) we get

and by $(2.1)$

$$
V=\left(Y^{\prime}\right)^{-1} I^{\prime},
$$

$$
E=A\left(A_{t} Y A\right)^{-1} A_{t} I .
$$

This is the fundamental formula for the solution of nodal networks by Kron's method.

5. Relation of the nodal theorem to the mesh theorem. For the given network let us consider any tree of the network. The voltage drops of the branches of the tree can be prescribed arbitrarily since there are no closed circuits on the tree to which Kirchhoff's mesh law applies. If we choose any node as $J_{1}$, construct $V$ as above (see sec. 2.1), define the voltage drop across any two nodes as the difference between the values of $V$ at the initial and terminal points of the node pair, and choose any sequence of nodes as defining a path, then Kirchhoff's mesh law will be satisfied for any closed path. Thus if we restore the branches, called chord branches, which were deleted to form the tree, then Kirchhoff's mesh law is certainly satisfied for meshes consisting of branch paths. Also if we replace these branches by their Thévenin equivalents then the currents in these branches which, as Synge has shown, are a complete set of mesh currents, are 
determined by Ohm's law. If we now replace the tree branches of the network by their Thévenin equivalents then by Synge's theorem all the branch currents are determined and hence all the branch voltage drops are determined. Conversely if we assign the chord branch currents in the network regarded as consisting of Thévenin branches, then by Synge's theorem, the branch currents and hence the branch voltages are determined. This implies that the branch voltage drops of the original network are determined. This completes the proof of the equivalence of the nodal and mesh methods.

In addition, if the Thévenin equivalent branches are given then in Synge's notation

$$
W=e-Z i \text { or } e=W+Z i
$$

where $e$ is the branch voltage-source matrix, $Z$ the branch impedance matrix and $i$ is the matrix of branch currents. In the notation of this paper if the Norton equivalent branches are given then by

$$
U=I-Y E .
$$

Since we are dealing with two representations of the same network we may identify $i$ and $U$, and $W$ and $E$. If $Z$ is non-singular (5.1) can be written

$$
i=Z^{-1} e-Z^{-1} E,
$$

and by comparison with (5.2) we have

$$
I=Z^{-1} e, Y E=Z^{-1} E .
$$

If we multiply (5.1) by $C_{t}$ on the left and note that $e^{\prime}=C_{t} e$ and $i=C i^{\prime}$ then we have

$$
e^{\prime}=C_{\imath} E+C_{t} Z C i^{\prime} .
$$

Since $e^{\prime}=C_{t} Z C i^{\prime}$ it follows that

$$
C_{\imath} E=0 .
$$

But by eq. (2.3) this may be written

$$
C_{t} A V=0 .
$$

Since $V$ can be prescribed arbitrarily we have

$$
C_{\imath} A=0 .
$$

This last relation can be derived on purely topological grounds. Also since in (5.3) $E$ is arbitrary we have

$$
Y=Z^{-1} .
$$

Equations (5.3), (5.4) and (5.5) show the relations of the two methods.

\section{BIBLIOGRAPHY}

1. W. H. Ingram and C. M. Cramlet, On the foundations of electrical network theory, Jnl. of Math. and Physics (23) 134-155(1944).

2. J. L. Synge, The fundamental theorem of electrical network, Quart. of Appl. Math., IX, 113-127 (1951).

3. P. Le Corbeiller, Matrix Analysis of Network, Harvard University Press, Cambridge, Mass., 1950, Chapter III.

4. O. Veblen, Analysis situs, Am. Math. Soc. Col. Pub., Vol. 5, Part II, 2nd ed., 1931, p. 68. 